\title{
ESTUDIO Y ANÁLISIS DE LOS MÉTODOS DE INICIACIÓN A LA VIOLA MÁS UTILIZADOS POR EL PROFESORADO DE LAS ENSEÑANZAS ELEMENTALES DE LA PROVINCIA DE VALENCIA
}

Ana María Botella Nicolás ${ }^{\mathbf{1}}$ : Universitat de València. España. ana.maria.botella@uv.es

Vicenta Fuster Martínez: Universitat de València. España. vicentafus17@gmail.com

\section{RESUMEN:}

El presente artículo estudia los métodos de iniciación a la viola más utilizados entre el profesorado de las Enseñanzas Elementales de la provincia de Valencia. Participan en el estudio 26 profesores de 21 conservatorios públicos. El instrumento de recogida de la información ha sido el cuestionario diseñado según criterios basados en la sencillez, precisión y concreción de los 32 ítems que lo componen y la discreción y preservación del anonimato en el manejo de la información recabada. La metodología de la investigación ha sido mixta. Se ha utilizado una estrategia de complementación que combina métodos de ambas orientaciones, cuantitativa y cualitativa, ocupándose las distintas perspectivas de una parcela diferente de la realidad que se quiere investigar. Se observa cómo el profesorado conoce gran variedad de métodos y libros de iniciación a la viola, pero contrariamente realizan ejercicios previos al uso del libro de iniciación. Por otra parte, los docentes reflejan que el método de iniciación a la viola más conocido y utilizado es el de Suzuki a pesar de que la mayoría de ellos no están formados en dicho método.

PALABRAS CLAVE: Viola - Enseñanzas elementales - Conservatorio - Investigación educativa - Profesorado - Métodos de iniciación - Valencia.

\footnotetext{
1 Ana María Botella Nicolás: profesora contratada doctora del Departamento de Didáctica de la Expresión musical, plástica y corporal de la Facultad de Magisterio de la Universitat de València.

Correo: ana.maria.botella@uv.es
} 


\title{
STUDY AND ANALYSIS METHODS VIOLA INTRODUCTION TO MORE USED BY TEACHERS OF ELEMENTARY EDUCATION IN THE PROVINCE OF VALENCIA
}

\begin{abstract}
:
This article studies the most commonly used methods of initiation viola among teachers in the elementary teachings of Valencia. In the estudy 26 teachers from 21 public conservatories are involved. The instrument of information collection the questionnaire has been designed as based on simplicity, accuracy and precision of the 32 items that compose and preservation of anonymity and discretion in handling information collected criteria. The research methodology has been mixed. We used a complementary strategy that combines both orientations methods, quantitative and qualitative, dealing with the different perspectives of a different part of reality to be investigated. It shows how the teacher knows a variety of methods and books for beginners viola, but contrary made use of the book prior to initiation exercises. Moreover, teachers show that the method of introduction to viola known and used is the Suzuki even though most of them are not trained in that method.
\end{abstract}

KEY WORDS: Viola - Basic teaching - Conservatory - Educational Research Teachers - Methods of initiation - Valencia.

\section{INTRODUCCIÓN}

La presente investigación trata sobre un tema poco estudiado de la didáctica musical y su praxis docente. Se basa en el estudio de los métodos de iniciación a la viola que utiliza el profesorado de los conservatorios de enseñanzas elementales de la provincia de Valencia. La investigación refleja la práctica docente en esta etapa y aborda otras cuestiones que hacen referencia al objetivo general que los docentes pretenden conseguir con su alumnado y a la metodología que utilizan para iniciarlos en la viola. También se tratan aspectos que resultan relevantes, como la preferencia de contenido de un libro de iniciación y los objetivos más trabajados en el primer curso de enseñanzas elementales.

La viola, hoy en día, aún es un instrumento poco conocido por la sociedad en general. Quizás es por esta razón por la que su estudio no tiene tanta demanda como el violín o el clarinete, por ejemplo. La viola ${ }^{2}$ es un eslabón fundamental dentro

\footnotetext{
${ }^{2}$ Pertenece al grupo de instrumentos de cuerda frotada. La viola que existe en la actualidad nace entre los siglos XVI y XVII, su tamaño es unos centímetros mayor que el violín. Dentro de las agrupaciones de música de cámara como el cuarteto de cuerda, o las orquestales, sirve de puente sonoro entre el violín y el violonchelo debido a su tesitura central. Se sostiene del mismo modo que el violín, con el brazo izquierdo en posición horizontal y en la caja armónica se apoya la barbilla. Con el brazo derecho se sujeta el arco. La viola tiene cuatro cuerdas que se afinan por quintas: Do, Sol, Re, La, de grave a agudo. Su lectura musical se realiza en clave de Do en tercera línea y, cuando la tesitura es muy aguda, en clave de Sol en segunda línea.
} 
de la familia del violín. A lo largo de la historia, ha pasado de ejercer un papel secundario a ser un instrumento solista e importante dentro de las agrupaciones: "La viola es un instrumento excepcional por su peculiar sonido, timbre y color, y se ha desarrollado como instrumento solista en obras de gran solidez desde el punto de vista técnico" (Vdóvina, 2006, p. 4).

La etapa de iniciación a un instrumento musical es un periodo muy importante y delicado; y más aún si se tiene en cuenta que, generalmente, son niños y niñas de entre 8 y 11 años quienes emprenden la aventura de iniciarse en el mundo de la música. Es en los cuatro primeros cursos donde se produce la evolución más rápida y notoria de toda la carrera de un músico. Así pues, el alumnado empieza por la familiarización con su instrumento y termina asumiendo unos contenidos básicos que le aseguran una base suficiente para afrontar las enseñanzas profesionales, en el caso de que así lo decida. Durante esta etapa, los alumnos se encuentran en continuo cambio respecto a su desarrollo físico, intelectual y emocional y como consecuencia de este hecho, necesitan una gradación muy especial de todos los conceptos, así como de su metodología.

Si bien se sabe de la importancia que tienen los cuatro cursos de enseñanzas elementales, cabe recalcar todavía más el primero de ellos, ya que es aquí donde se forjan las bases para el posterior desarrollo de una técnica instrumental. Por ello, los docentes han de ser minuciosos y muy conscientes de su papel en la iniciación a un instrumento musical. En estos primeros momentos, es cuando los profesores juegan un roll decisivo a la hora de marcar unas pautas que luego quedarán impresas durante todo el camino musical del alumno:

La epistemología de la práctica para el dominio de un instrumento musical está centrada en la práctica de un aprendizaje tutorizado en el aula, más la praxis docente no necesariamente ha sido fundamentada en la reflexión. Lo que ha predominado es una racionalidad técnica en la que el docente simplemente presiona al estudiante a aplicar un conocimiento teórico en su práctica instrumental (Matos, 2006, p. 69).

En la mayoría de casos, los docentes tienen claros los objetivos que se pretenden alcanzar y los contenidos a desarrollar, pero muchas veces se deja de lado la reflexión en torno a la práctica en el aula. Es decir, se conocen bien los conceptos técnicos pero no se hace una reflexión sobre cómo transmitir los conocimientos al alumno. Según Matos (2006, p. 65), "es necesario cambiar la concepción y el modus operandi, de quienes se dedican a enseñar un instrumento musical'.

Por otra parte, es necesario contribuir a aumentar la literatura sobre la didáctica de la Viola dado el vacío que existe en este campo. Para Muñiz (2013, p. 343): "Históricamente la viola, como especialidad instrumental, ha sido tratada de manera secundaria". También, desde el punto de vista pedagógico, la viola ha estado durante muchos años a la sombra del violín. Con el paso del tiempo, este instrumento ha ido adquiriendo independencia "gracias a los estudios reglados, a una metodología específica, al interés de los compositores en la creación de un nuevo 
repertorio y a la aparición de los grandes solistas, entre otros factores" (Muñiz, 2012, p. 10). No obstante, la literatura existente sobre la viola en términos de bibliografía o investigación sigue siendo escasa.

\subsection{Consideraciones sobre la iniciación a un instrumento musical}

La iniciación a un instrumento musical forma parte de la Educación Musical. Para Frega este concepto se puede definir como:

En su doble aspecto de educación y de música, la Educación Musical es un proceso de enseñanza-aprendizaje que, partiendo de las posibilidades sensorio auditivas de los educandos y de sus posibilidades expresivas por la voz y la ejecución instrumental, crea situaciones de aprendizaje de amplio espectro, ayudando al sujeto en su proceso de cognición, ejercicio y valoración de este lenguaje artístico, promoviendo inclusive su elección vocacional en los casos de capacidades específicas evidentes (1997, p. 7).

En opinión de Willems (2001, p. 31): "En la actualidad, todavía es común confundir la educación musical con el estudio de una técnica instrumental'. Y, en este punto, es imprescindible establecer las diferencias entre la iniciación musical y la iniciación instrumental:

La educación musical propiamente dicha se sitúa más allá de toda aplicación instrumental y concierne a los elementos fundamentales de la música: sentido rítmico, oído musical, sentido melódico, nombres de las notas, grados de la escala, improvisación, conocimientos armónicos (Willems, 2011, p. 188).

Según explica Carrascosa en su tesis (2006), la iniciación musical tiene como finalidad introducir a aquellas personas que deseen comprender y manejar el lenguaje musical, en la forma receptiva y expresiva. Willems (2011) afirma que la iniciación a la música no pertenece al campo de la pedagogía musical, sino que está relacionada con la educación general de los niños. Es decir, son las personas del entorno familiar las que por medio de canciones de cuna y populares, sonajeros, juegos rítmicos y movimientos, introducen a los pequeños en la música. Incluso desde el vientre materno. $Y$, por otro lado:

La verdadera iniciación instrumental constituye más bien una meta específica de cierto tipo de iniciación musical. Aquella que tiene como objeto introducir al alumno en el conocimiento y manejo de las estructuras básicas del lenguaje musical a través de un determinado instrumento (Hemsy de Gainza, 1993, p. 10).

Cuando se habla del proceso de enseñanza instrumental, se trata de didáctica aplicada a la enseñanza de un instrumento musical. En un principio, la didáctica se definió como el arte de enseñar. Esta definición daba a entender que la acción del docente venía dada por un don innato. Mucho después se reconoció la base científica 
en la enseñanza. En el siglo pasado, ya se centró el interés en el educando en vez de en el educador. Según Palacios (1998, p. 1), "la didáctica es la acción que el docente ejerce sobre la dirección del educando, para que éste llegue a alcanzarlos objetivos de la educación". Este proceso de aprendizaje necesita de una serie de medios y recursos técnicos.

Como afirma Swanwick (1991, p. 83), "todos somos potencialmente musicales, como todos somos potencialmente seres capaces de adquirir el lenguaje; pero eso no significa que el desarrollo musical pueda darse sin estimulación". Por tanto, las capacidades musicales necesitan ser incitadas, al igual que sucede con la adquisición del lenguaje. Si se pretende una enseñanza de calidad, deben respetarse los principios didácticos o leyes del aprendizaje que propone Palacios (1998):

1. Proximidad: La enseñanza parte del punto más cercano posible a la vida del alumno. Utilizando elementos familiares, como por ejemplo canciones populares previamente conocidas por el alumnado, o ejercicios amenos y relacionados con su entorno.

2. Ordenamiento, causalidad y contigüidad espacial y temporal: Para que el aprendizaje sea eficaz, se debe seguir una secuencia lógica a la hora de seleccionar las tareas y los conocimientos que deben aprender, de modo que las ideas sean fáciles de asociar y asimilar. Los métodos de enseñanza deben organizarse de lo simple a lo complejo, de esta forma, lo aprendido en una lección o pieza se consolida y amplia en la siguiente.

3. Adecuación: La materia se debe adaptar a las necesidades y posibilidades de cada estudiante, así como también de la sociedad.

4. Participación: La mejor forma de aprendizaje es aquella que implica la experiencia del alumno. Todo aprendizaje instrumental ya es de por sí un proceso en el que el educando se mantiene activo, pero se puede incrementar la participación del alumno obligando a que cante previamente aquello que va a tocar, por ejemplo.

5. Espontaneidad: Este apartado hace referencia a la creatividad. Muchos de los métodos existentes dejan poco margen al fomento de la creatividad en el alumno. Si se sigue un método, lo más probable es que los objetivos principales sean que el alumno primeramente escuche lo que va a tocar $y$, seguidamente, que lo ejecute ciñéndose al máximo posible a la partitura.

6. Actividad y frecuencia: Tal y como expresa Schumann (1955, p. 28) "Diligencia y perseverancia son los factores más importantes del progreso". Se debe de realizar un esfuerzo por aprender, basándose en la repetición y en la constancia del trabajo en casa.

7. Autocorrección: Con el tiempo, los alumnos deben de ser capaces de reconocer los problemas técnicos e interpretativos que se les presenten, y adquirir la capacidad de solucionarlos sin ayuda.

8. Eficiencia: Se trata de obtener el mejor resultado con el mínimo esfuerzo. Este concepto está relacionado con una correcta posición del cuerpo respecto con el instrumento, y la realización de los movimientos de acuerdo con las leyes naturales del movimiento. Invirtiendo el tiempo de estudio de una manera inteligente. 
9. Efecto: Fijando pequeñas metas que se puedan ir superando a corto plazo se consigue motivar al alumnado.

10. Motivación: Una materia por sí misma no tiene la capacidad de generar su aprendizaje, es el método utilizado por el profesor el que la hace rica e interesante. "El método por sí solo no es garantía de una enseñanza eficaz, pues para serlo, necesita ser vitalizado y dinamizado por la personalidad del docente" (Serna, 1985, p. 46). Un profesor que no cuide y ponga en constante renovación su método puede llegar a conseguir que su alumnado aborrezca la asignatura. Según las palabras de Neuman (2004, p. 9): "/os docentes necesitan renovar sus modelos de actuación y, en muchos casos, reencauzar e incluso recuperar la ilusión y el entusiasmo necesarios para desarrollar su actividad profesional" (...). "La figura del maestro adquiere una importancia fundamental por la gran riqueza de intercambios que se establecen con el alumno". Es por ello que la motivación al alumnado debe ir acompañando al método de cada profesor, así como el entusiasmo por enseñar y aprender.

11. Emoción: En el estudio de la música, al igual que sucede en el estudio de todas las artes, el educando consigue dominar una serie de conceptos y habilidades; pero además, se muestran los propios sentimientos y se descubren los de los demás por medio de la interpretación.

Cabe diferenciar la enseñanza instrumental para los niños que se inician y la enseñanza instrumental para alumnos con una formación previa como ejecutantes (Palacios, 1998). En el caso de la iniciación, es la primera vez que el niño toma contacto con el instrumento. Mientras que en el segundo, el niño ya viene con una base instrumental más o menos desarrollada. Los docentes se pueden encontrar ante ambos casos, pero este trabajo se centra en los aspectos relacionados con alumnos que experimentan su primera vivencia instrumental. Métodos como el de Suzuki o el de Paul Rolland, se basan en los principios didácticos o leyes del aprendizaje expuestos anteriormente. Ambos métodos pretenden hacer accesible a los niños el aprendizaje de un instrumento, teniendo en cuenta su edad psíquica y fisiológica.

Willems (1981, p. 47) afirma que "en la educación musical, por lo general, se han descuidado los primeros pasos". Durante mucho tiempo se generalizó la creencia de que existía un don innato para la música y su aprendizaje. Y los niños no dotados estaban condenados al fracaso. En la educación que proponen Willems y otros autores del s. XX (Kodály, Orff, Dalcroze), se le otorga mucha importancia a los primeros pasos, es decir a la iniciación, y no se distingue a los alumnos por sus capacidades o dotes. En la iniciación musical e instrumental es fundamental el contacto con los niños. En esta etapa, de tres a seis años de edad, los pequeños se encuentran en el "periodo de desarrollo de las afectividades sensoriales y emotivas" (Willems, 1981, p. 65). Es por ello que la afectividad jugará un papel importante tanto en este tiempo como a lo largo de toda la educación musical.

El problema viene cuando "la mayoría de los profesores de instrumento nunca han recibido entrenamiento en el área de la pedagogía" (Palacios, 1998, p. 3). Según indica el Forum delle Associazioni disciplinari della scuola (1999, pp. 11-15), los 
docentes deben adquirir conocimientos y competencias en los siguientes registros: "el saber experto y el saber enseñado, el estudiante, la relación y la comunicación, la evaluación, los colegas, los padres, recursos externos respecto a la escuela". Así mismo, Willems (1981, p. 47) sostiene que además se "exige del profesor la posesión de las bases psicológicas de la educación musical" y "ser capaz de encarar una educación desde el principid". Por lo tanto, ya no es suficiente que el docente sepa tocar a la perfección un instrumento, sino que cada vez más, se pretende que sea un verdadero educador (Jorquera, 2002).

\subsection{Aproximación a la didáctica de la viola}

Muñiz (2013) explica que la viola, como especialidad instrumental, ha sido tratada de forma secundaria. La ausencia de un currículo propio como especialidad, dentro de los conservatorios, es una de las principales razones por las que se ha dado esta situación a lo largo de la historia. El estudio de viola obtuvo reconocimiento y autonomía a partir del siglo XIX, cuando la Cátedra del Conservatorio de Bruselas contrató, de forma pionera, un profesor de viola en 1877 (Lainé, 2010). En España, esa diferenciación no queda bien establecida hasta mediados del siglo XX. Fue el Decreto del 10 de septiembre de 1966, que trata sobre la reglamentación general de los Conservatorios de Música, el que conllevó una profunda reorganización de los estudios musicales en España y afectó directamente a la enseñanza de la viola (Agüeria, 2011). La enseñanza de este instrumento se separa de la del violín, tanto en conservatorios profesionales como superiores:

Esta circunstancia nos impide hablar de una escuela pedagógica violística antigua, con su propia metodología desarrollada, que tenga en cuenta las características sonoras del instrumento, su rol dentro de la familia de cuerda y las particularidades técnicas específicas que la diferencian del violín (Muñiz, 2013, p. 344).

No obstante, existen varios métodos y libros de estudios para viola publicados previamente a la implantación de la especialidad de viola en los conservatorios europeos. En referencia a la producción española de métodos para el instrumento, destacan los siguientes: Método elemental de viola y nociones generales de la viola de amor (1870), Estudio de la viola: Siete ejercicios difíciles (1874) y el Método sobre la viola de amor de siete cuerdas (1894), escritos por Tomás Lestán. Más tarde, José María Beltrán compone los Doce estudios o caprichos de mediana dificultad (1881). Y el guitarrista Graciano Tarragó, publica en 1954 su Método graduado para el estudio de la viola. Así como también, resalta la obra para viola de Francisco Fleta Polo (1931), gran pedagogo que compuso diversos estudios y obras hechas a la medida de sus estudiantes. Ya en los años 1980 y 1990, el violista y pedagogo Emilio Mateu realizó diversas publicaciones como son: La Viola, Iniciación (1986) que obtuvo el premio del MEC a la Edición; La viola: escalas y arpegios, con ejercicios preparatorios y complementarios (1988) y La Viola: Iniciación a la Música, Volumen 1 y 2 (2006).

Tal y como apunta Palacios (1998, p. 7), "no hay una didáctica musical general, sino que dependerá del profesor, y también del alumno. Lo cual demuestra la gran 
importancia que tiene la preparación del docente en el área de la ejecución instrumental'. Y no solo en la ejecución del instrumento, sino que ha de poseer una adecuada formación en el campo de la pedagogía musical. Porque también, se puede "desaprobar el objetivo del profesor si tiende conscientemente hacia el virtuosismo y no hacia la musicalidad" Willems (2001, p. 32). Este autor entiende por musicalidad el ajuste y la sucesión de la cultura musical, el desarrollo auditivo, solfeo e instrumento. $Y$ por virtuosismo se concibe el desarrollo de unas habilidades en el dominio de la técnica de instrumental que deslumbran al público. Según la tradición, el proceso de enseñanza - aprendizaje en la música se ha centrado en el contenido y no en el alumno. Es decir, que se ha procurado inculcar a los estudiantes la técnica básica que se necesita para ejecutar con gran dominio un instrumento musical. Pero, "se requiere un cambio en la didáctica que oriente, guíe y cree condiciones para que el alumno logre sus objetivos, le genere un aprendizaje significativo y lo capacite para construir su propio conocimiento" (Matos, 2006, p. 67). Para que ese cambio pueda darse, es necesario que el profesorado reflexione a cerca de sus prácticas educativas. "En la praxis, la reflexión es de gran valor puesto que le permite al docente mejorar su actuación y visualizar la relación entre la teoría y la práctica. El educador reflexivo no nace, se hace" (Matos, 2006, p. 77).

Por tanto, un docente ha de tener claros una serie de conceptos antes de iniciar su labor. Es decir, ha de reflexionar y elaborar su propia metodología. Para ello, ha de definir sus intenciones educativas, tener conocimiento de las bases psicopedagógicas y ser consciente del contexto educativo en el que está.

\section{OBJETIVOS}

En la presente investigación se plantea como objetivo principal conocer cuáles son los métodos de iniciación a la viola más utilizados entre el profesorado de los Conservatorios gestionados por la Consellería de Educación en la provincia de Valencia. De éste se desprenden los siguientes objetivos específicos:

1. Descubrir las metodologías de iniciación a la viola que utilizan los docentes.

2. Invitar a los profesionales en la materia a la reflexión docente.

3. Contribuir a aumentar la literatura de la didáctica de la viola.

\section{METODOLOGÍA}

Para alcanzar los objetivos propuestos se han utilizado los siguientes procedimientos metodológicos:

1. Una revisión bibliográfica que se hace analizando las fuentes primarias y secundarias sobre lo que hasta el momento se ha escrito en relación a la iniciación a un instrumento musical. Y más concretamente, aquello referente a la iniciación a la viola.

2. Un estudio que hace referencia a la praxis de la viola en las aulas de los centros elementales de música de la provincia de Valencia. Mediante el "Cuestionario para conocer los Métodos de Iniciación a la Viola más utilizados por el profesorado de los conservatorios de Enseñanzas 
Elementales en la provincia de Valencia", se ha conocido la actitud del profesorado, y ha permitido a los docentes expresar su punto de vista acerca de la mejor manera de enseñar este instrumento y cuáles son los pilares en los que basan la iniciación que dan a los alumnos.

\subsection{Poblaciones y muestras.}

La población de referencia está compuesta por el conjunto de profesores de viola de los conservatorios de enseñanzas elementales de la provincia de Valencia. Los centros públicos objeto de estudio han sido 22 con un total de 26 docentes (24 mujeres y 2 hombres).

\subsection{Procedimiento de recogida de la información.}

A partir del diseño metodológico de esta investigación, que combina la necesidad de obtener información cuantitativa y cualitativa en relación con los objetivos planteados, la recogida de información se efectuó a través de los siguientes procedimientos:

1. Revisión, vaciado y análisis de revistas, documentos legales, tesis doctorales y publicaciones de investigación.

2. Revisión, vaciado y análisis de los principales métodos de iniciación a un instrumento de cuerda, tales como Suzuki y Rolland.

3. Revisión vaciado y análisis de los aspectos fundamentales a tener en cuenta tanto en la iniciación musical como en la iniciación instrumental, así como también cuestiones referentes a la didáctica de la viola y selección y la adecuación del método a seguir.

4. El cuestionario que es el documento que más información ha proporcionado. Está formado por 32 ítems y una batería de preguntas abiertas y cerradas. Está dividido en 3 dimensiones.

La primera categoría corresponde a la caracterización de la muestra, en ella se abordan variables de clasificación como el sexo, edad, nacionalidad y centro y cargo en el centro ( 5 ítems). La segunda dimensión hace hincapié en el perfil del profesor, encuestando la experiencia docente, sus estudios y su formación pedagógica (15 items). El tercer apartado pretende averiguar qué métodos de iniciación a la viola conocen, qué tipo de ejercicios realizan o qué métodos utilizan en sus clases, qué aspectos consideran más importantes a la hora de elegir un libro de iniciación para su alumnado y observaciones (12 ítems). El cuestionario ha sido validado a través de un contraste de opiniones con expertos que han verificado que los ítems propuestos están en consonancia con los objetivos de la investigación, siguiendo así con el criterio de triangulación. También ha sido sometido a diferentes criterios de validez de contenido, de constructo y de criterio. En su elaboración se han tenido en cuenta los siguientes puntos:

- La definición del constructo a medir.

- $\quad$ El propósito de la escala.

- La composición de los ítems (número, contenido, definición y ordenación). 
- La prevención de los sesgos en su cumplimentación y codificación de las respuestas.

\subsection{Técnicas de análisis de datos.}

Las siguientes categorías clasifican los análisis realizados con la información obtenida de las diferentes fuentes:

1. Análisis documental de la información: revistas, libros, estatutos, a través de la revisión, vaciado y el análisis posterior.

2. Análisis descriptivos de los datos obtenidos sobre las variables de información y de clasificación incluidas en el cuestionario (frecuencias, porcentajes y medias). Para realizar este análisis se ha utilizado el programa estadístico SPSS 18.0.

3. Análisis relacionales para comprobar el grado de asociación entre variables significativas.

4. Análisis de contenido de los datos cualitativos obtenidos mediante las preguntas abiertas del cuestionario.

\section{DISCUSIÓN}

Resalta el hecho de que la mayoría de sujetos son mujeres y solamente dos de ellos son hombres. En lo que a la edad se refiere, cabe señalar que la mayoría de profesores se encuentran entre los 40 y 49 años. Coincide que todos los docentes son de nacionalidad española y que ninguno de ellos ocupa cargo directivo en el centro donde imparten docencia.

Se observa que el $50 \%$ de los profesores lleva de 15 a 20 años dedicándose a la docencia de la viola. Otro dato que destaca es que el $88,89 \%$ de los sujetos realizaron sus estudios superiores en la Comunidad Valenciana, en las provincias de Valencia y Castellón, frente a un docente que los realizó en Madrid. Este dato induce a pensar que la movilidad del profesorado es casi inexistente, es decir, que estudiaron en Valencia y, posteriormente, trabajan también allí. Otro aspecto a comentar es que solo el $37,5 \%$ del profesorado obtiene formación pedagógica sobre los instrumentos de cuerda y su didáctica. El resto de docentes no contestan, no obtienen formación, o la obtienen de forma indirecta. Respecto a la variable donde se pregunta si se dedican a la investigación en el campo de la docencia, resulta que el $37,5 \%$ contesta que sí. Este hecho contrasta con que ninguno de los sujetos posee la suficiencia investigadora, o no contestan a esta pregunta. Por otro lado, el $75 \%$ de los docentes afirma asistir a cursos o seminarios de profesorado. En contraposición, el $75 \%$ de los profesores afirman no impartir cursos o seminarios.

En cuanto a los objetivos generales que pretenden conseguir en el alumnado, los profesores han derivado en dos posiciones: el dominio de aspectos técnicos de la viola y el desarrollo del interés hacia la música a través de la viola.

Se corrobora que el profesorado es conocedor de varios libros que sirven para el inicio del aprendizaje a este instrumento. No obstante, el más utilizado es el de 
Suzuki (35,71\%). Cabe explicar que este libro no empieza desde un nivel cero, es decir, que para poder abordar la primera de las piezas del primer volumen, es necesario el conocimiento de ciertos aspectos teóricos y, sobre todo, técnicos. Este hecho se debe a que la filosofía Suzuki contempla una serie de ejercicios previos que no están recogidos en las páginas del libro. String Builder y Sapmannhaus fue anotado por 2 sujetos, y obtuvieron un porcentaje del $14,29 \%$ cada uno. Rolland, Sheila Nelson, La viola, Mi amiga la viola y Waggon Wheels los utiliza un profesor, y cada uno de estos métodos obtiene un 7,14\%.

Los profesionales afirman que utilizan y siguen los contenidos de un libro desde el principio con los alumnos que se inician en un $75 \%$. Sin embargo, también aseguran que realizan ejercicios previos al uso de éste. El profesorado nombró un total de 9 ejercicios que realizan con sus alumnos previamente al uso de un libro. Los más comunes fueron los de sujeción de la viola y el arco (21,43\%). Seguidamente, los ejercicios posturales, los de pre-lectura, la enseñanza de la clave de Do, y los de formación de la mano izquierda obtuvieron un porcentaje del 14,29\% cada uno. Los CD's, las escalas, los ejercicios de paso de arco con cuerdas al aire, ejercicios de pizzicato y las charlas para padres fueron nombradas por el $7,14 \%$ de los docentes.

Estas dos cuestiones, seguir los contenidos de un libro desde el principio y realizar ejercicios previos entran en contradicción, ya que si sostienen que realizan ejercicios previos es porque el libro no los considera. Generalmente, se necesitan una serie de ejercicios, prácticas y explicaciones antes de poder ejecutar con éxito cualquier pieza. No parece que el origen sea relevante a la hora de que un docente elija un libro, pero sí que resultaron más votados los de procedencia española, japonesa y alemana con un $18,75 \%$. Los profesores aseguraron que su elección estaba basada fundamentalmente en la secuenciación de los contenidos y en el propio conocimiento de la aplicación del mismo. Algunos de ellos explicaron que utilizan más de uno en función de lo que cada estudiante necesite.

Cuando se les preguntó qué métodos españoles recomendarían para guiar a los principiantes, Mi amiga la viola $(26,67 \%)$ de Luis Roig y La viola $(20 \%)$ de Pablo García fueron los más señalados. Ambas son publicaciones hechas por docentes de la provincia de Valencia. También hubo sujetos que citaron algunos que no eran de origen español, pese a que en la cuestión estaba especificada esta característica. Con un 13,33\%, Stradivari fue mencionado por 2 profesores. El resto de libros recomendados, como: La viola de Francisco Serra, Nícolo, Susy Sentamans, Emilio Molina y Escalas y arpegios de Emilio Mateu, lo fueron solo por 1 profesor cada uno, lo que supone un $6,67 \%$. Otra respuesta fue la imposibilidad de recomendar ningún libro o método por no aportar ningún tipo de innovación.

Los dos objetivos del primer curso de Enseñanzas Elementales que el profesorado consideró más importantes fueron la adecuada posición del cuerpo respecto al instrumento $(15,28 \%)$ y el conocimiento de las partes de la viola $(13,54 \%)$. Estos dos ítems son fundamentales en un primer contacto con el instrumento ya que, desde el inicio han de tenerse muy en cuenta los aspectos posturales que permitan el correcto desarrollo de la técnica violística. De lo contrario, una mala posición 
podría derivar en problemas técnicos e incluso en lesiones musculares. Por otra parte, el conocimiento de las partes del instrumento es una cuestión básica, considerando primordial que el alumno sepa qué tiene en sus manos para después poder enseñarle su manejo. En menor medida el profesorado consideró los siguientes objetivos: el mecanismo de la mano izquierda, la coordinación entre ambas manos, el desarrollo del interés por la música y el cuidado del instrumento, con 12,85\% respectivamente. La adquisición de un hábito de estudio diario $(12,50 \%)$, el trabajo en las cuatro cuerdas $(11,46 \%)$ y el funcionamiento correcto del arco (8,68\%). Es de lógica pensar que este último sea el menor votado ya que la destreza en ambas manos no se espera hasta cursos posteriores.

Respecto a las características que se tienen en cuenta para elegir un libro, el contenido de melodías y canciones populares conocidas por el alumno es la más apreciada por los profesionales. Es una manera de acercar el aprendizaje a los estudiantes, ya que al conocer las piezas que se van a trabajar pueden diferenciar fácilmente aspectos de afinación y métrica, además de ser una forma de motivación el hecho de llegar a tocar ellos mismos canciones que conocen. Uno de los docentes realizó una aportación interesante, indicando que podrían incluirse historias atractivas y modos de autoevaluación por parte del alumno.

De los análisis relacionales, uno de los resultados a destacar es que, a mayor experiencia docente, tienden a valorarse más los aspectos que contribuyen en términos musicales a un desarrollo integral de los estudiantes. El interés por la música y el cuidado del instrumento es uno de los puntos que más se aprecia, independientemente del objetivo general de cada profesor. Del mismo modo ocurre con la adquisición de un hábito de estudio diario.

Otro dato que se observa es que el profesorado que utiliza mayor variedad de ejercicios y material didáctico es aquél que ha recibido formación pedagógica. Este hecho parece razonable ya que al recibir formación específica se conocen y se amplían metodologías y recursos para utilizar en el aula.

Finalmente, se percibieron dos grandes tendencias entre el profesorado respecto al objetivo general a conseguir con sus estudiantes. Por un lado, están aquellos que pretenden despertar el interés por la música a través de la viola. Y por otro, los que buscan que se dominen los aspectos técnicos del instrumento. Las dos visiones son interesantes y complementarias. Es cierto que en la etapa de iniciación se procura despertar interés y amor por la música, y que el mejor vehículo para ello es el aprendizaje de un instrumento. Pero también deben tenerse en cuenta aspectos técnicos que permitan tocarlo con naturalidad y destreza. Si bien no se pretende formar virtuosos de la viola, sí que se intenta formar músicos que se sientan cómodos tocando las piezas que se le presentan en el repertorio, y para ello resulta imprescindible el dominio de la técnica. De lo contrario, un alumno que tenga muchas dificultades técnicas podría no llegar a disfrutar e incluso con el tiempo perder el interés en la música.

\section{CONCLUSIONES}


Dentro del proceso de la Educación Musical, la etapa de la iniciación instrumental es una de las más complejas e importantes por varios factores. El primero de ellos está ligado a la corta edad del alumnado y, por tanto, hay que considerar sus capacidades físicas, motoras y cognitivas. Para iniciarse en el manejo de un instrumento musical se han de tener en cuenta algunos aspectos que están relacionados con la consciencia corporal, como la posición del instrumento respecto al propio cuerpo, la sujeción del instrumento en ambas manos o el esfuerzo corporal que se está ejerciendo. Así como también, tienen que conocerse y, posteriormente, aplicarse una serie de conocimientos teórico-prácticos sobre el funcionamiento del instrumento que se está aprendiendo y, a su vez, relacionarlos con los conocimientos adquiridos previamente del lenguaje musical. Este aprendizaje significativo puede ser complejo cuando los alumnos no terminan de dominar conceptos del lenguaje musical y necesitan estos mismos para empezar la lectura de las partituras. En esta etapa inicial, se necesita mucha paciencia y dedicación por parte del profesorado, de los padres y del propio alumno. No obstante, lo importante es detacar el principio que muchos pedagogos han defendido a lo largo de la historia de la Educación Musical y que recuerda que todos los seres humanos son potencialmente musicales. Es decir, que nacen con las capacidades necesarias para aprender música, y que para ello se necesitan estímulos correctos y una guía adecuada.

Por lo tanto, conviene que el docente esté respaldado por una formación pedagógica que le permita desempeñar su labor con efectividad y destreza. El profesor tiene que hacer accesible y ameno el aprendizaje del instrumento. Para ello, el docente necesitará diseñar y seguir un método que le permita acercar los contenidos al alumnado, manteniéndole motivado. En este punto, la figura del profesor es fundamental para que el proceso de enseñanza-aprendizaje se lleve a cabo con éxito, ya que se convierte en una figura modelo para el alumno. La actitud del docente influenciará en gran medida al futuro musical del aprendiz, ya que depende del enfoque que se le dé a la iniciación se puede conseguir despertar el interés por la música o todo lo contrario. Si lo que se pretende es estimular el amor por la música mediante el aprendizaje de un instrumento musical, se requerirá creatividad, imaginación, afecto y formación que permita enfrentarse a esta tarea de una forma consciente. En estos primeros pasos se debe procurar generar una atracción por la música y, por supuesto, por el instrumento elegido. Si bien es cierto que tampoco pueden descuidarse los aspectos básicos que fundamenten una técnica correcta para el futuro dominio del instrumento.

Existen métodos, como Suzuki (1969, 1981, 1993, y 2004) o Rolland (1991), que aportan su visión filosófica de la iniciación al violín y la viola, y que a su vez establecen unas directrices para su desempeño. Ambas filosofías apoyan que todo niño, estimulado y guiado correctamente, puede llegar a tocar con destreza el instrumento. Y están pensados para acercar el aprendizaje del violín, en este caso, a cualquier alumno que lo desee.

Por su parte, el método diseñado por Suzuki pretende utilizar la enseñanza del instrumento como medio para formar a los niños en personas con valores y de 
corazón noble. Para ello, utiliza lo que él mismo llamó el "Método de la lengua materna", que introduce el aprendizaje de la viola de la misma forma que se adquiere el habla (Suzuki, 1969). Sus características más importantes son: la importancia del período temprano, el ambiente en el que se desarrolla el niño, la participación de los padres en la formación musical, la figura de un docente experto, la práctica y la constancia, el respeto por las diferencias personales y el afecto.

Por otro lado, Rolland (1991) diseñó un proyecto que tenía como finalidad crear instrumentistas capaces de tocar utilizando movimientos coordinados y sin exceso de tensión muscular. Este pedagogo trató de solventar diferentes problemas técnicos en la ejecución del violín que derivan de una incorrecta gestión de la tensión-relajación de los músculos. Su método se centra en la postura del estudiante, en el movimiento, y en la felicidad del alumno cuando aborda un repertorio en el que se siente cómo en el manejo del instrumento.

Respecto a los objetivos que se planteaban al principio, cabe destacar que el primero de ellos, donde se pretendía conocer cuáles son los métodos de iniciación a la viola más utilizados entre el profesorado de viola de los Conservatorios gestionados por Consellería en la provincia de Valencia, se han cumplido. La información recibida ha resultado de gran utilidad para conocer una parte de la realidad actual en este campo. Se pudo averiguar que el profesorado conoce gran variedad de métodos y libros de iniciación a la viola, y que los más destacados resultaron ser el método de Suzuki, el método de Rolland y Mi amiga la viola de Luis Roig Cerveró. Sin embargo, en la práctica, los métodos que más se utilizan son Suzuki, String Builder y Sapmannhaus.

El segundo objetivo propuesto, estaba enfocado en descubrir las metodologías de iniciación a la viola que utilizan los docentes. La mayoría afirmó que, desde un principio, utiliza un libro como guía para el alumno, y las razones se basaron en que de esta forma resulta más fácil el seguimiento y la secuenciación de los contenidos para el alumno, y la comodidad del libro frente a llevar otro tipo de material u hojas sueltas. Contrariamente, también confirmaron que realizan ejercicios previos al uso del libro de iniciación, tales como: ejercicios posturales, charlas para padres, ejercicios de sujeción de la viola y del arco, ejercicios de pre-lectura para enseñar la clave de Do, ejercicios de pizzicato, ejercicio de paso de arco con cuerdas al aire, ejercicios de formación de la mano izquierda, uso de CD'S, y escalas.

Respecto a los dos últimos objetivos presentados, se puede decir que sí se han cumplido ya que esta investigación invita a los profesionales de la materia a la reflexión docente y a la continua búsqueda de recursos y estrategias que ayuden a mejorar la labor del profesorado.

Finalmente las conclusiones más destacadas que se extraen de este trabajo son las siguientes: En primer lugar, podemos decir que el profesorado posee, en general, bastante experiencia docente. Los análisis parecen indicar que aquellos profesores que han recibido formación poseen mayor variedad de recursos y estrategias que los que no la recibieron. Solo tres han recibido formación pedagógica sobre la didáctica 
de los instrumentos de cuerda. Esto puede deberse a varias razones: que en su época de estudiantes no se les exigía formación pedagógica para ejercer como docentes o que ellos mismos no lo consideran importante. Un dato a resaltar es que dos de los que sí tienen formación pedagógica, se especializaron en el Método Suzuki, y puede ser porque actualmente existe una tendencia en realizar este tipo de formación, ya que están en auge las academias basadas en este método. Un hecho que contrasta con que la mayoría de docentes no recibieron formación pedagógica es que estos mismos indican que sí acuden a seminarios o cursos de profesorado.

El método de iniciación a la viola más conocido y utilizado por el profesorado es el de Suzuki. Esto puede resultar paradójico, cuando la mayoría de ellos no están formados en dicho método. Es necesario señalar que, para ejercer como profesor Suzuki, se necesita una formación específica e ir obteniendo niveles certificados. Así como también debe tenerse en cuenta que los conservatorios públicos no ofertan éste método como tal. Se podría decir que, el método Suzuki se utiliza en las aulas de los conservatorios, desligado de su filosofía y de su esencia. Es decir, que el profesorado sigue la secuenciación y el repertorio pero no sigue los pasos de este método ni su filosofía. El método Suzuki fue publicado en 1978. La versión que se comercializa en España está escrita en inglés. Por tanto, el anexo final, en el que se explica la forma correcta de ejecutar cada pieza, es complicado de entender por parte del joven estudiante. Cuenta con acompañamiento de piano, pero no está incluido en mismo libro del alumno, sino que hay que comprarlo aparte. Actualmente, viene acompañado con CD. El repertorio está graduado progresivamente en su nivel de dificultad. Incorpora melodías populares de otros países, así como también piezas clásicas, como minuetos de J. S. Bach, etc. Se contemplan ejercicios cortos que trabajan aspectos técnicos previos a cada lección, y algunas escalas como Re Mayor, Sol Mayor y Do Mayor.

Los profesionales se decantaron en libros de origen español, alemán y japonés. Entre las razones de sus preferencias, la más importante fue la secuenciación de contenidos. Lo que hace pensar que, probablemente, los libros de iniciación españoles, alemanes y japoneses estén mejor secuenciados, según la opinión del profesorado. Cuando se les preguntó qué métodos de iniciación a la viola españoles recomendaría, Mi amiga la viola de Luis Roig y La viola de Pablo García fueron los más nombrados por los docentes. Por una parte, Mi amiga la viola, publicado en 2003, es un libro de melodías populares adaptadas a la iniciación de la viola. También cuenta con la partitura del acompañamiento de piano para cada una de las piezas. Cabe señalar que enuncia los objetivos propios de cada lección. Cuenta con la letra de todas las canciones y con un pequeño dibujo que hace referencia al contenido de ésta. Excepto las tapas, está impreso en blanco y negro.

Por otra parte, La viola de Pablo García es un libro más reciente, publicado en 2012. El interior está impreso en escala de grises y las tapas a color. Este libro también incluye canciones populares con acompañamiento de piano, dúos de viola y un CD. Está organizado por unidades y en cada una se contemplan una serie de ejercicios preparatorios que facilitan la posterior ejecución de las piezas. Para hacer más 
atractivo este método, Violeta, que es la mascota, ofrece consejos, frases de motivación, recordatorios y breves nociones de lenguaje musical.

Las características que más aprecian los docentes en un libro de iniciación son, en primer lugar, que contenga canciones y melodías populares conocidas por el alumno. $Y$, seguidamente, que éstas dispongan de acompañamiento al piano o a dúo con el profesor. También valoran el que se incluya un $C D$, para que el alumno tenga la oportunidad de escuchar las piezas que va a tocar, e incluso que las pueda tocar junto con la reproducción del $C D$, ya que facilita el aprendizaje de las mismas y es más ameno. Por el contrario, el aspecto por el que menos se decantan a la hora de elegir un libro es que realice breves apuntes de lenguaje musical. Uno de los profesionales realizó un apunte y anotó que le gustaría que se incluyeran historias que resultaran atractivas y modos de autoevaluación para el alumno. Esta aportación resultó interesante ya que habitualmente los métodos no suelen incluir autoevaluaciones y puede resultar útil para el propio estudiante. De este modo, se daría cuenta de su evolución, de las cosas que debería mejorar y, a su vez, sería una herramienta de motivación personal.

Una de las preguntas incluidas en el cuestionario pedía que se esbozara una visión general de aquello que cada docente pretendía conseguir con su alumnado, y las opciones más consideradas fueron: despertar el interés por la música a través de la viola y dominar los aspectos técnicos de la viola. Estas dos vertientes son bastante lógicas ya que en esta primera etapa se pretende acercar al estudiante a la música, utilizando como vehículo la viola. Y por otro lado, es necesario tener cierto dominio de la técnica del instrumento si se pretende ejecutar con éxito el repertorio propuesto. Las perspectivas de futuro profesional en este campo también fueron consideradas, pero en menor medida. Este hecho podría deberse a que el alumnado todavía está en una fase muy temprana y las metas propuestas deben ser a corto plazo.

Algunos de los profesionales hicieron observaciones sustanciosas al final del cuestionario, y mostraron su implicación e interés en este tema. En primer lugar, uno de ellos quiso establecer la diferencia entre método entendido como una filosofía global de abordar la iniciación a un instrumento, y el método o libro que se utiliza para el seguimiento de los contenidos y el repertorio que tiene dominar el alumno.

Otro profesor, recalcó la importancia que tiene la implicación de los padres en la iniciación instrumental y desarrollo musical del niño. Explicó que, en su caso, invita a los padres a asistir a las clases, para que tomen nota de las explicaciones $y$, hasta incluso, hagan fotografías o graben vídeos para trabajar mejor determinados conceptos cuando practiquen en casa. También aclaró que, aparte del libro, recomienda a sus alumnos que lleven una libreta y que anoten todo aquello que se ha trabajado y los ejercicios que tienen que estudiar para la próxima sesión. Esta observación resulta muy beneficiosa, ya que en la actualidad los niños asisten a multitud de actividades extraescolares, tales como deportes o idiomas, además de asistir a clases de música. En algunos casos, la asistencia a este tipo de actividades es para mantener a los más pequeños ocupados mientras los padres cumplen con su 
horario laboral. Esto deriva en que realmente no se ponga mucho interés en el verdadero progreso musical. Para que verdaderamente exista un desarrollo y un avance es necesaria la implicación de los padres, ya que, además, el niño se compromete en mayor medida cuando ve que los padres también lo están.

En la actualidad, son muchos los jóvenes que se decantan por el estudio de la viola. $\mathrm{Y}$, año tras año, aumenta progresivamente el número de principiantes. Esta investigación contribuye a incrementar el repertorio didáctico de este instrumento e invita a todos los docentes de la materia a reflexionar en torno a su práctica docente. Los datos extraídos en el presente estudio son interesantes para aquellos que estén interesados en la didáctica de la viola, ya que no se conocen trabajos precedentes similares.

\section{REFERENCIAS}

Agüeria, F. (2011). Historia de la educación musical en la España contemporánea. Un estudio de política legislativa. Oviedo: FA.

Carrascosa, F. (2006). Estudio descriptivo-comparativo de métodos de iniciación pianística. (Tesis inédita de doctorado). Universidad Nacional de San Juan, Argentina.

Forum delle Associazioni disciplinari della scuola (1999). Formazione dei docenti: riflessioni e proposte. Musica Domani, 112, 11-15.

Frega, A. (1997). Metodología comparada de la Educación Musical. (Tesis inédita de doctorado). Collegium Musicum, Buenos Aires.

Hemsy de Gainza, V. (1993). La iniciación al piano. Notas al margen del pentagrama, en Revista latinoamericana de Educación Musical, 2(5), 10-14.

Jorquera, M. (2004). Métodos Históricos o Activos en Educación Musical, en Revista Electrónica Léeme, 1-55. Recuperado de http://musica.rediris.es/leeme/. Consultado el 3/12/2014.

Lainé, F. (2010). L'alto. Bressuire. France: Anne Fuzeau Productions.

Matos, R. (2006). La práctica de la reflexión durante el aprendizaje de un instrumento musical, en Revista de Investigación, 59, (65-85).

Muñiz, L. (2012). La viola en España: Historia de su enseñanza a través de los principales métodos y estudios. (Tesis inédita de doctorado). Universidad de Oviedo, España. 
Muñiz, L. (2013). Principales aportaciones a la didáctica de la viola de Francisco Fleta Polo: El nuevo sistema para el estudio de las posiciones fijas, en DEDiCA: Revista de educaçao e humanidades, 4, 343-354.

Neuman, V. (2004). La formación del profesorado y los conciertos didácticos. Profesorado, en Revista de currículum y formación del profesorado, 8 (1), 1-12. Recuperado de http://www.ugr.es/ recfpro/rev81ART6.pdf. Consultado el 28/14/2015.

Palacios, M. (1998). La Didáctica aplicada a la enseñanza de los instrumentos, en Revista Electrónica Léeme, 1-7. Recuperado de http://musica.rediris.es/leeme/revista/palacios98.pdf. Consultado el 4/3/2015.

Rolland, P. (1991). L'Enseigment du mouvement dans le jeu de les cordes: techniques formatives et correctives pour le violon et alto. Canada: Les Presses de I'Université Laval.

Serna, A. (1985). El método didáctico, en Educación Física y Deporte, 7 (1-2), 42-46. Recuperado de http://aprendeenlinea.udea.edu.co/revistas/index.php/educacionfisicaydeporte/arti cle/viewFile/4679/4112. Consultado el 4/3/2015.

Schumann, R. (1955). Consejos a los jóvenes estudiantes de música. Buenos Aires: Ricordi Americana.

Suzuki, S. (1969). Ability Development from Age Zero. Miami: Warner Bros. Publications Inc.

Suzuki, S. (1981). Ability Development from Age Zero. USA: Alfred Publishing Co., Inc.

Suzuki, S. (1993). Shinichi Suzuki: His Speeches and Essays (About Suzuki). USA: Alfred Publishing Co., Inc.

Suzuki, S. (2004). Educados con amor. El Método Clásico de la Educación del Talento. USA: Alfred Publishing Co., Inc.

Swanwick, K. (1991). Música, pensamiento y educación. Madrid: Morata.

Vdóvina, M. (2006). La viola en el conjunto instrumental y su desarrollo individualizado. (Tesis inédita de doctorado). Facultad de Música. Instituto Superior de Arte, La Habana.

Willems, E. (1981). El valor humano de la Educación Musical. Barcelona: Editorial Paidós. 
Willems, E. (2001). El oído musical: La preparación auditiva del niño. Barcelona: Editorial Paidós.

Willems, E. (2011). Las bases psicológicas de la educación musical. Barcelona: Editorial Paidós.

\section{AUTOR/ES:}

\section{Ana María Botella Nicolás:}

Doctora en pedagogía por la Universitat de València. Es Licenciada en Geografía e Historia, especialidad Musicología y maestra en Educación Musical, por la Universidad de Oviedo. Grado profesional en la especialidad de piano. Durante el año 2001 obtiene por oposición plaza en el cuerpo de profesores de música de enseñanza secundaria en Alicante (actualmente en excedencia). Ha presentado diversas comunicaciones en jornadas y congresos sobre didáctica de la música así como distintas publicaciones. Es profesora contratada doctora del departamento de didáctica de la Expresión Musical, Plástica y Corporal de la Facultad de Magisterio de la Universitat de València. Forma parte de la Comisión de Coordinación Académica del Master Universitario en Profesor/a de enseñanza secundaria de la UVEG y del Máster de Investigación en didácticas específicas. Desde Febrero de 2015 dirige el aula de música.

Orcid: 0000-0001-5324-7152 y ResearchID: N-1375-2014.

\section{Vicenta Fuster Martínez:}

Titulada superior de viola por el Conservatorio Superior de música Joaquín Rodrigo de Valencia. Es Máster Universitario en Profesor/profesora de Educación Secundaria de la Universitat de València y Máster en Investigación en Didácticas específicas, especialidad de educación musical por la UVEG. En la actualidad prepara su tesis doctoral en el campo de la didáctica de la viola. 